Bolm Inst. oceanogr., S Paulo, 34 (ünico):71-77, 1986

\title{
CONTRIBUIÇÃO AO CONHECIMENTO DA BIOLOGIA DA MANJUBA, Anchoviella lepidentostole (FOWLER, 1911), NO ESTUÄRIO DE SÃO VICENTE, SP (OSTEI CHTHYES, ENGRAULIDAE)
} Alfredo Martins PAIVA FILHO; Maria de Lourdes ZANI-TEIXEIRA* \& Paulo Kiyoshi
KIHARA**

Instituto Oceanogrä́ico da Universidade de São Paulo (Caixa Postal 9075, 01051 São Paulo, SP)

\section{Synopsis}

As many as 3408 specimens from 16 samples were collected in São Vicente Estuary, São Paulo, Brazil, from June 1979 to July 1980. The occurrence, growth, length-weight relationship and condition factor of the species are analysed. This estuarine region is one of the areas where the species grows, moving from it when the maturation process begins.

Descriptors: Growth, Length-weight relationships, Condition factor, Estuaries, Anchoviela lepidentostole, Engraulidae, São Vicente: SP, Channel of the Barreiros.

Descritores: Crescimento, Relação peso/comprimento, Fator de condição, Estuärios, Anchoviella lepidentostole, Engraulidae, São Vicente: $\mathrm{SP}$, Canal dos Barreiros.

\section{Introdução}

A manjuba Anchoviella lepidentostole è atualmente, da família, a espécie de maior importância comercial no sudeste do Brasil, não sendo porém a única, uma vez que Anchoa januaria é bastante comercializada em Paranaguá (PR) e Engraulis anchoita estā se tornando importante para a pesca na região, quando de suas migrações vindas de águas argentinas.

A. lepidentostole ocorre das Guianas até o Estado do Paranā, sendo que nos meses quentes é capturada em grandes quantidades na região do Rio Ribeira de Iguape, no Estado de São Paulo (Figueiredo \& Menezes, 1978).

No Estuārio de São Vicente é capturada o ano todo, sendo a produção destinada à preparação do Irikô, alimento apreciado notadamente pela colônia japonesa do Estado. A pesca é realizada no Canal dos Barreiros (Fig. 1) com o emprego de

(*) Pös-graduanda da ärea de oceanografià biolögica.

(**) Estagiário no Departamento de Oceanografia Biolögica.

Publ. n. 653 do Inst. oceanogr. da Usp.

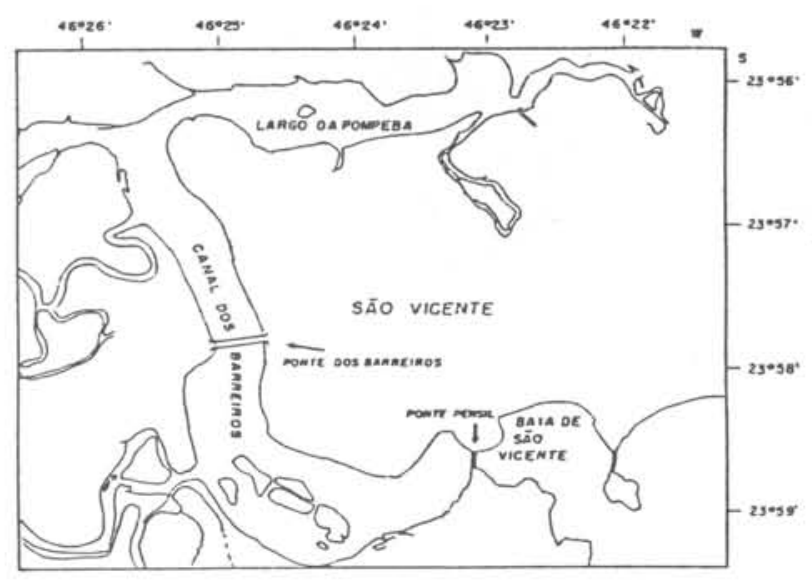

Fig. 1. Mapa da região estudada mostrando a ponte dos Barreiros, sobre o canal de mesmo nome, onde foram coletados especimens de Anchoviella lepidentostole.

rede especial, localmente conhecida como "jaú" e mostrada na Figura 2.

Neste trabalho são analisados a ocorrência, o crescimento, a relação peso/comprimento e o fator de condição da espécie. 


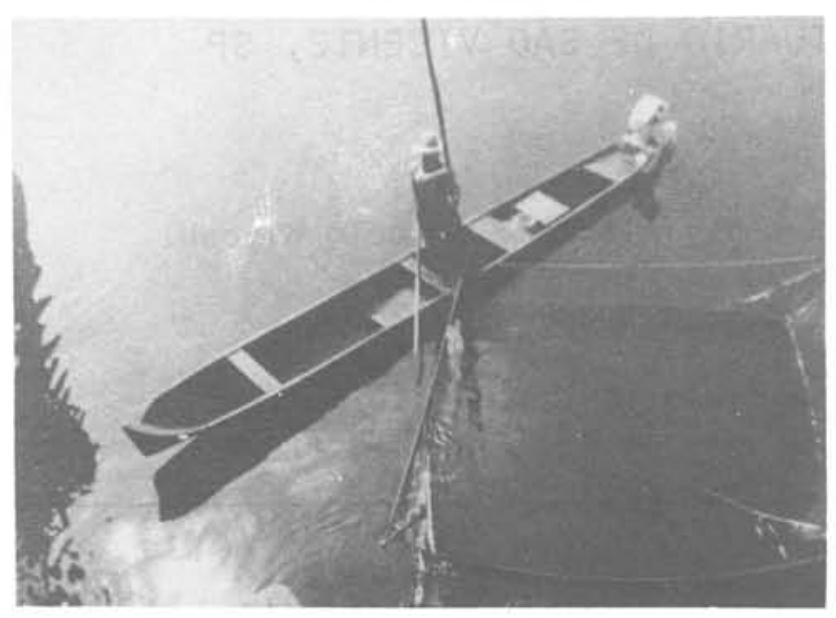

Fig. 2a. Operação de pesca da rede "jaū". Pescador: Saburo Morota.

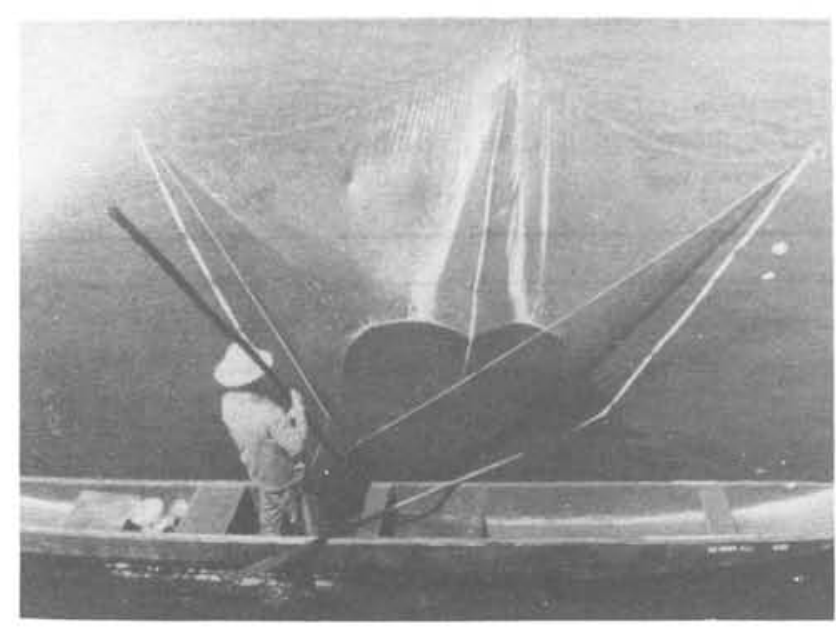

Fig. 2b. Operação de pesca da rede "jaü". Pescador: Saburo Morota.

\section{Material e métodos}

0 material analisado consta de dezesseis amostras coletadas no período de junho/79 a julho/80, num total de 3408 exemplares.

As amostras foram provenientes de capturas realizadas pelos pescadores locais, nas proximidades da ponte dos Barreiros (Fig. 1). As capturas foram executadas por contrato de modo a possibilitar a manutenção das características do aparelho de pesca, o local de captura e o acompanhamento direto das operações de pesca.
Do total capturado, em cada pescaria, retirou-se uma amostra ao acaso de aproximadamente 200 exemplares, dos quais, em laboratório, foram tomados os seguintes dados: a) comprimento: considerado como a distância, em milímetros entre a ponta do focinho e a extremidade da cauda ligeiramente distendida; b) peso: em grama, utilizando-se uma balança tipo Welsh com aproximação atē decigrama; c) sexo, e d) estádio de maturidade, segundo escala de quatro estádios descrita em Vazzoler (1971)。

Curvas de distribuição de freqüência de comprimento foram construídas para cada amostra (Fig. 3), utilizando-se classes de $1 \mathrm{~mm}$ de amplitude.

Para obtenção das curvas de crescimento,os dados de comprimento médio dos exemplares de cada amostra foram lançados em gráfico contra o tempo (Fig. 4).

Os dois grupos mostrados nas Figuras 3 e 4, oriundos certamente de dois lotes de desova, que ocorreram, respectivamente, nos períodos de junho até dezembro/79 e de fevereiro até julho/80, fizeram com que passasse-se a proceder à análise dos dados em separado para cada grupo, denominados doravante I e II, respectivamente.

Estabeleceu-se para cada grupo a relação peso/comprimento (Fig. 5), a partir dos dados de peso médio por classe de $2 \mathrm{~mm}$ de comprimento (Tab. 1) sendo, para efeito de ajuste das curvas, considerado o valor do ponto médio da classe $(54,95 \mathrm{~mm} ; 56,95 \mathrm{~mm}$; etc.). Segundo Hayashi \& Kondo (1962) é preciso muita atenção, quando do uso da equação alométrica para expressar a relação peso/comprimento, porque os coeficientes "b" e a constante "a" variam de acordo com a amplitude de comprimento considerado, razão pela qual, neste estudo, só foram considerados exemplares que estivessem dentro da mesma amplitude de comprimento (Fig. 5) para que, eliminando a influência do comprimento, os resultados dos grupos I e II fossem passiveis de comparação.

Calculou-se o fator de condição $K$ (Le Creen, 1951) para cada exemplar amostrado e o $\mathrm{K}$ médio $(\overline{\mathrm{K}})$ por amostra. Tendo em vista que o $\mathrm{K}$ freqüentemente pode sofrer influencia do comprimento (Le Creen, op. cit.), anteriormente à obtenção do $\overline{\mathrm{K}}$ por amostra (Tab. 2), procedeu-se ao cálculo do mesmo apenas para exemplares da classe de comprimento de 

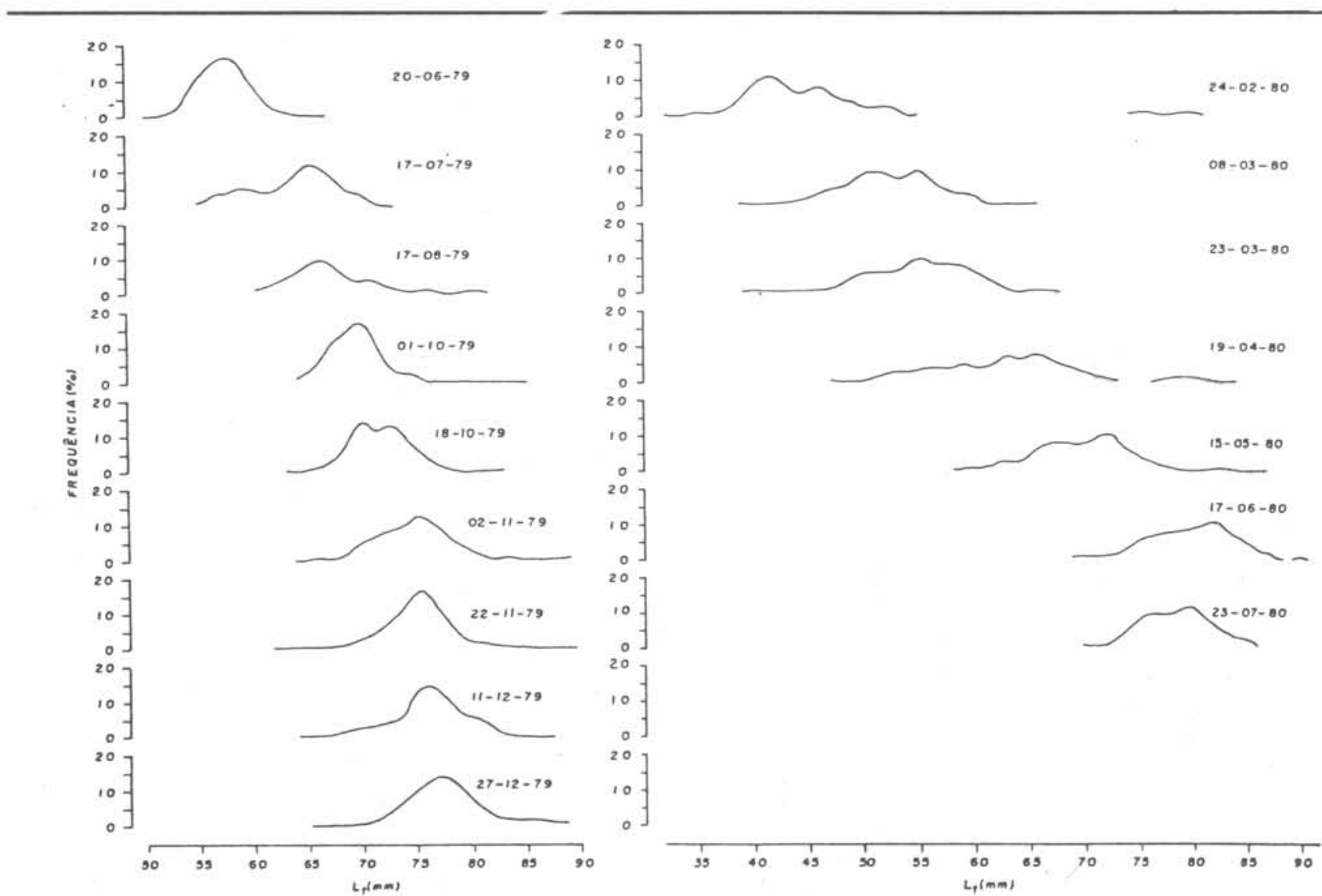

Fig. 3. Distribuição de freqüência de comprimento por coleta de Anchoviella lepidentostole, Estuärio de São Vicente, SP.

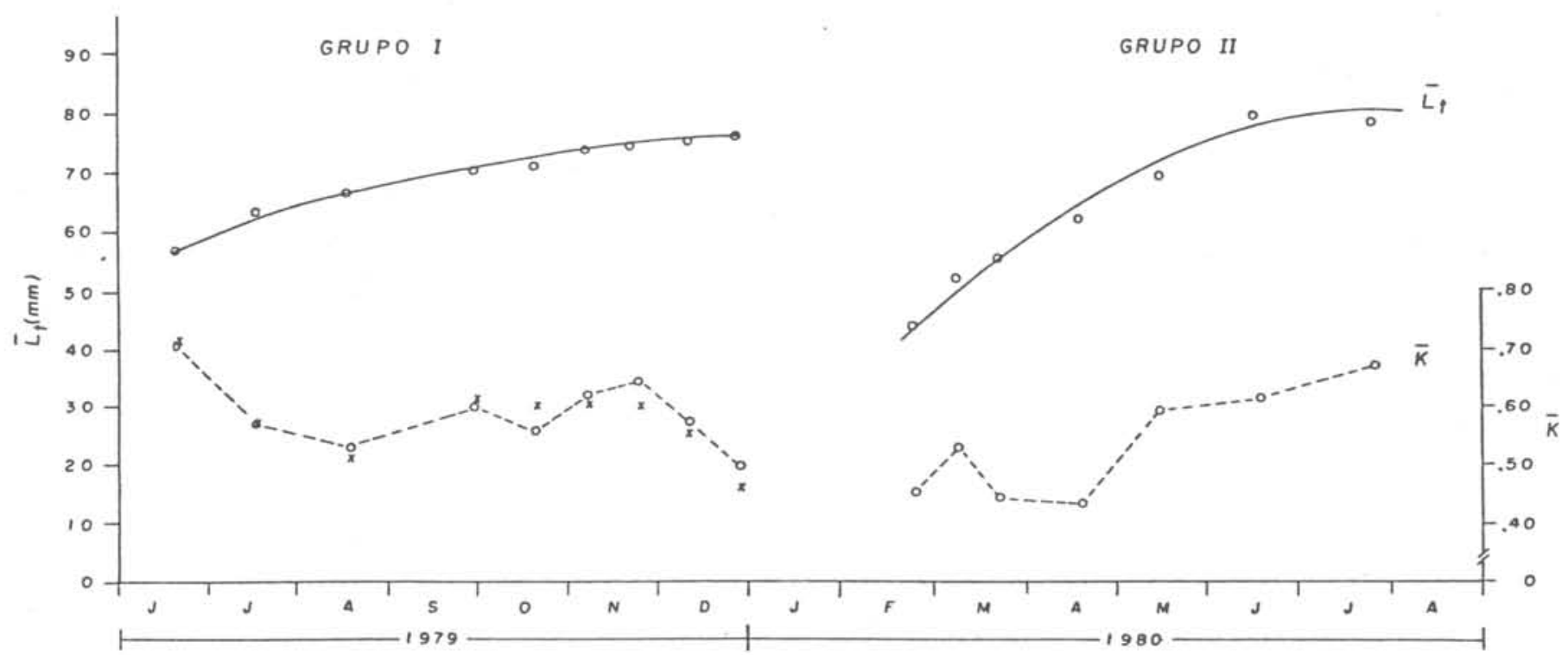

Fig. 4. Anchoviella lepidentostole, Estuärio de São Vicente: curvas de crescimento (- $)$, e valores mëdios do fator de condição por amostra $(----)$; assinalados por $(x)$ os valores calculados apenas para a classe de comprimento de 63 a $65 \mathrm{~mm}$. 


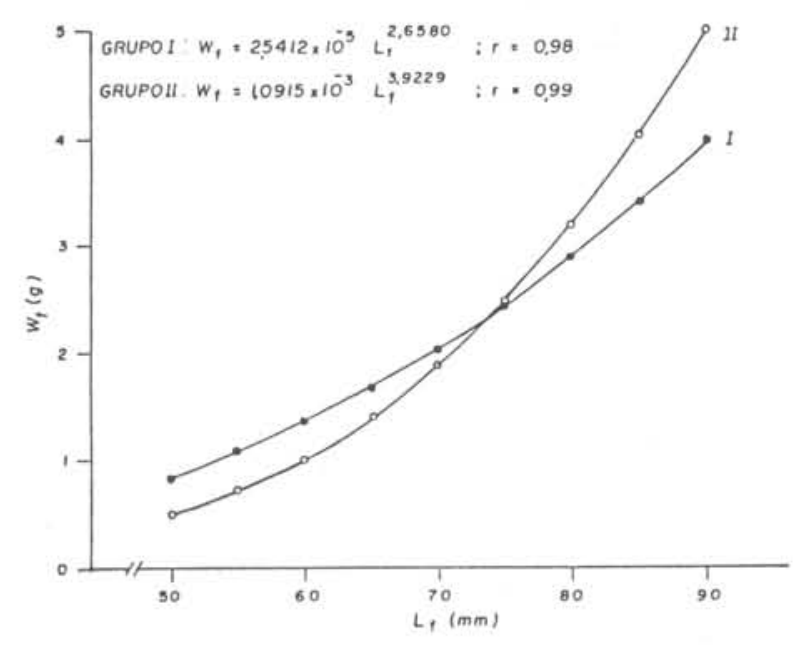

Fig. 5. Relação peso/comprimento de Anchoviella lepidentostole, Estuärio de São Vicente.

Tabela 1. Dados de peso mëdio por classe de comprimento onde $\mathrm{L}_{\mathrm{t}}=$ classe de comprimento $(\mathrm{mm}), W_{\mathrm{t}}=$ peso mëdio $(\mathrm{g})$, SX $=$ desvio da mëdia e $n=$ nümero de exemplares na classe, para Anchoviella lepidentostole, Estuärio de São Vicente.

\begin{tabular}{|c|c|c|c|c|c|c|}
\hline & & GRUYO 1 & & & Ruro II & \\
\hline Lt & $\bar{w}$ & $5 \bar{x}$ & $n$ & $\overline{w t}$ & $5 \bar{x}$ & n \\
\hline $541-56$ & 2,1881 & 0,0387 & 42 & 0,7429 & 0,0186 & 98 \\
\hline $561-58$ & 1,3075 & 0,0605 & 80 & 0,8263 & 0,0262 & 76 \\
\hline 38160 & 1,3589 & 0.0254 & 90 & 0,9521 & 0.0324 & 71 \\
\hline $60 \longmapsto 62$ & 1,3452 & 0,0380 & 62 & 1,0491 & 0.0378 & 53 \\
\hline $621-64$ & 1,4016 & 0,0358 & 62 & 1,1810 & 0,0495 & 58 \\
\hline${ }^{64} 上^{66}$ & 1,5416 & 0.0246 & 113 & 1,5022 & 0.0592 & 45 \\
\hline${ }^{66} \longmapsto^{68}$ & 1.6877 & 0,0208 & 138 & 1,5522 & 0.0436 & 67 \\
\hline $681-70$ & 1.8621 & 0,0244 & 145 & 1,6918 & 0,0414 & 49 \\
\hline $70 \vdash 3$ & 2,0370 & 0,0209 & 216 & 2,0254 & 0.0546 & 59 \\
\hline $721-74$ & 2,2710 & 0,0252 & 221 & 2,4197 & 0,0528 & 61 \\
\hline $741-76$ & 2,4730 & 0,0227 & 252 & 2,6912 & 0,0437 & 68 \\
\hline $76 \vdash 78$ & 2,5520 & 0.0259 & 227 & 2,7940 & 0,0408 & 83 \\
\hline $781-80$ & 2.7114 & 0,0378 & 223 & 3,1231 & 0,0418 & 79 \\
\hline $801-82$ & 2,9566 & 0,0471 & 76 & 3,3631 & 0.0379 & 84 \\
\hline $82 \mid-84$ & 3,2357 & 0,0737 & 28 & 3,5716 & 0,0320 & 76 \\
\hline${ }^{84} j-86$ & 3,6640 & 0,0966 & 25 & 3,9000 & 0.0586 & 42 \\
\hline $86 \longmapsto-88$ & 4,0125 & 0,1297 & 16 & 4,1267 & 0.0746 & 15 \\
\hline
\end{tabular}

63 a $65 \mathrm{~mm}$, presentes em quase todas as amostras do grupo I, estando os resultados apresentados na Tabela 3 e assinalados na Figura 4.

Considerando-se os valores de comprimento médio e peso médio, este último calculado a partir das equações representativas das relações peso/comprimento, e o intervalo de tempo, em dias,
Tabela 2. Fator de condição mëdio por amostra, onde $\bar{K}=$ fator de condição mẻdio, $S \bar{x}=$ desvio da média, $\mathrm{n}=$ nümero de exemplares e $\mathrm{NA}=$ nümero da amostra, para Anchoviella lepidentostole no Estuärio de São Vicente, SP.

\begin{tabular}{ccccc}
\hline NA & Data & $\overline{\mathrm{k}}$ & $\mathrm{s} \overline{\mathrm{x}}$ & $\mathrm{n}$ \\
\hline 01 & $20 / 06 / 79$ & 0,71 & 0,0084 & 224 \\
02 & $17 / 07 / 79$ & 0,57 & 0,0046 & 222 \\
03 & $17 / 08 / 79$ & 0,53 & 0,0061 & 227 \\
04 & $01 / 10 / 79$ & 0,60 & 0,0037 & 210 \\
05 & $19 / 10 / 79$ & 0,56 & 0,0064 & 220 \\
06 & $07 / 11 / 79$ & 0,62 & 0,0052 & 200 \\
07 & $22 / 11 / 79$ & 0,64 & 0,0048 & 210 \\
08 & $11 / 12 / 79$ & 0,57 & 0,0038 & 210 \\
09 & $27 / 12 / 79$ & 0,49 & 0,0049 & 220 \\
10 & $24 / 02 / 80$ & 0,45 & 0,0093 & 220 \\
11 & $08 / 03 / 80$ & 0,53 & 0,0044 & 220 \\
12 & $21 / 03 / 80$ & 0,44 & 0,0049 & 220 \\
13 & $18 / 04 / 80$ & 0,43 & 0,0112 & 220 \\
14 & $15 / 05 / 80$ & 0,59 & 0,0082 & 220 \\
15 & $17 / 06 / 80$ & 0,61 & 0,0042 & 220 \\
16 & $23 / 07 / 80$ & 0,67 & 0,0044 & 200 \\
& & & & \\
\hline
\end{tabular}

Tabela 3. Fator de condição mëdio por amostra, apenas para os exemplares da classe de comprimento de 63 a $65 \mathrm{~mm}$, onde: $\overline{\mathrm{K}}=$ fator de condição mëdio, $s \bar{x}=$ desvio da média e $\underline{n}=$ nümero de exemplares. $\mathrm{NA}^{-}=$nümero da amostra.

\begin{tabular}{rlccr}
\hline NA & Data & $\overline{\mathrm{K}}$ & $\mathrm{S} \overline{\mathrm{x}}$ & $\mathrm{n}$ \\
\hline 01 & $20 / 06 / 79$ & 0,72 & 0,0413 & 7 \\
02 & $17 / 07 / 79$ & 0,57 & 0,0059 & 130 \\
03 & $17 / 08 / 79$ & 0,50 & 0,0079 & 114 \\
04 & $01 / 10 / 79$ & 0,61 & 0,0069 & 76 \\
05 & $19 / 10 / 79$ & 0,60 & 0,0240 & 27 \\
06 & $07 / 11 / 79$ & 0,61 & 0,0313 & 5 \\
07 & $22 / 11 / 79$ & 0,60 & 0,0409 & 7 \\
08 & $11 / 12 / 79$ & 0,56 & 0,0146 & 7 \\
09 & $27 / 12 / 79$ & 0,46 & 0,0180 & 4 \\
\hline
\end{tabular}

transcorrido entre a data de coleta de uma amostra e sua subseqüente, calculou-se os valores das taxas de incremento em comprimento e peso, conforme mostram as Tabelas 4 e 5 . Nestes cálculos, devido ao curto espaço de tempo decorri- 
do entre uma amostra e outra, admitiu-se como isométrica a relação, para efeito do cálculo das taxas.

\section{Tabela 4. Taxas de incremento em com- primento $(\mathrm{mm} / \mathrm{dia})$, onde: $\overline{L t}=$ comprimento mëdio por amostra, $d=$ diferença dos comprimentos de amostras subseqüentes, $t=$ número de dias entre uma amostra e ou- tra, $d / t=$ taxa de incremen- to, $\overline{d / t}=$ taxa mëdia de in- cremento e $\bar{S} \bar{x}=$ desvio da média.}

\begin{tabular}{|c|c|c|c|c|c|c|c|}
\hline & GRUPO & I & & & GRUPO & II & \\
\hline$\overline{1 . t}$ & d & $t$ & $d / t$ & $\overline{L t}$ & d & t & $d / t$ \\
\hline 37.087 & 6,28 & 27 & 0,2326 & 43.66 & 8,47 & 13 & 0,6515 \\
\hline $63.36<$ & 3.26 & 31 & 0.1052 & 52,132 & 2,77 & 13 & 0,2131 \\
\hline $66,62 \zeta$ & 2,58 & 45 & 0,0573 & $54,90 \angle$ & 7,13 & 28 & 0,2546 \\
\hline $69,20<$ & 2,10 & 18 & 0,1167 & $62,03 \mathrm{~L}$ & 7,24 & 27 & 0,2681 \\
\hline $71,30<$ & 3,34 & 19 & 0,1758 & 69.274 & 10,09 & 33 & 0,3058 \\
\hline 74.64 & - & 15 & - & 79,36 & - & - & - \\
\hline 76,097 & 1,21 & 19 & 0,0637 & 77,93 & - & - & - \\
\hline $75.30<$ & 1,21 & 16 & 0,0756 & - & - & - & - \\
\hline $76,31 /$ & - & - & - & - & - & - & - \\
\hline
\end{tabular}

$\bar{d} 7 t=0,11815 \bar{x}-0,0244$

$\bar{d} / t=0,3386 \quad s \bar{x}=0,0796$

Tabela 5. Taxas de incremento em peso (g/dia), para Anchoviella lepidentostole no Estuário de São Vicente, SP, onde $\overline{\mathrm{Lt}}=$ comprimento mëdio por amostra (mm), $\overline{W t}=$ peso médio da amostra $(\mathrm{g})$, estimado a partir de $\overline{L t}$, $d=$ diferença de peso de amostras subseqüentes, $\mathrm{t}=$ nümero de dias entre uma amostra e outra, d/t $=$ taxa, $\overline{d / t}=$ taxa média e $S \bar{x}=$ desvio da média.

\begin{tabular}{|c|c|c|c|c|c|c|c|}
\hline$\overline{L_{t}}$ & $\overline{w_{t}}$ & d & $t$ & $d / t$ & $\overline{d / t}$ & $s \bar{x}$ & \\
\hline 57.08 & $1,1851$. & 0,3790 & 27 & 0,0140 & & & \\
\hline 63,36 & 1,5641 & 0,2231 & 31 & 0,0072 & & & c \\
\hline 66.62 & 1,7872 & 0,1899 & 45 & 0.0042 & & & R \\
\hline 69,20 & 1.9771 & 0,1636 & 18 & 0,0091 & $0,0088 \pm$ & 0,0015 & v \\
\hline 71,30 & 2,1607 & 0.2769 & 19 & 0,0146 & & & $p$ \\
\hline 74.64 & 2,4176 & - & 15 & - & & & o \\
\hline 74.09 & 2,3706 & 0,1073 & 19 & 0,0036 & & & \\
\hline 75.30 & 2,4749 & 0,1071 & 16 & 0.0067 & & & I \\
\hline 76,51 & 2,5820 & - & - & - & & & \\
\hline 43,66 & 0.2964 & 0,2979 & 13 & 0.0229 & & & $c$ \\
\hline 52,13 & 0,5943 & 0,1338 & 13 & 0.0103 & . & & R \\
\hline 54,90 & 0,7281 & 0,4474 & 28 & 0,0160 & $0,0223 \pm$ & 0,0048 & $v$ \\
\hline 62.03 & 1.1755 & 0.6372 & 27 & 0.0236 & & & $\mathbf{P}$ \\
\hline 69,27 & 2,8127 & 1,2775 & 33 & 0.0387 & & & 0 \\
\hline \multirow[t]{2}{*}{79,36} & 3,0902 & - & - & - & & & \\
\hline & & & & & & & II \\
\hline
\end{tabular}

\section{Resultados e discussão}

\section{Ocorrência}

A. lepidentostole, dentro do período abrangido por este estudo, ocorreu nos meses de junho a dezembro de 1979 e de fevereiro a julho de 1980 , não sendo turada nos meses de janeiro e agos to de 1980 (embora diversas tentativas tenham sido feitas). Pelo exame da Figura 3 nota-se claramente para o grupo I, que os indivíduos da espécie penetraram no estuārio representados certamente por exemplares constituintes de ummesmo lote de desova, devido ao tipo de distribuição de frequência de comprimento apresentada.

0 grupo I apresentou exemplares com comprimento variando de 50 a $60 \mathrm{~mm}$, os quais permaneceram no estuário atẻ atingirem de 64 a $92 \mathrm{~mm}$, enquanto que para o grupo II os valores observados foram de 32 a $54 \mathrm{~mm}$ e deixaram o estuário ao atingirem comprimentos variando de 70 a $86 \mathrm{~mm}$. Observa-se, ainda, na Figura 3 a ocorrência em fevereiro/80 de poucos exemplares com comprimentos ao redor de $80 \mathrm{~mm}$, remanescentes do grupo I.

0 comportamento das distribuições de freqüência de comprimento (Fig. 3) com o evidente deslocamento das modas e o afastamento do grupo I, provavelmente para o mar, a partir de janeiro/80, e penetração do grupo II em fevereiro/80, fez com que se concluisse que coortes da espécie utilizam o estuário de são Vicente como uma das āreas de crescimento.

A penetração de jovens no estuārio ocorreria no verão, após o período de desova.

Observou-se a penetração de jovens do grupo II no estuário, o que não foi possivel para o grupo I por falta de observaçoes em meses anteriores a junho/79. O grupo I seria formado pelos remanescentes da desova anterior que teriam penetrado no estuário no último verão. Por essa hipótese os peixes do grupo I deveriam ser maiores que os maiores do grupo II. A diferença poderia ser atribuída a condições ambientais diferentes nesses dois anos, afetando o crescimento de ambos os grupos. Em termos de idade o grupo I seria formado por indivíduos mais velhos que os do grupo II o que explicaria a taxa de crescimento baixa e o início da maturação sexual apresentada, como mostrado a seguir. Nessas condições o grupo I seria como 
que uma continuidade do grupo II, mantidas as óbvias diferenças em comparação direta.

\section{Crescimento}

As curvas de crescimento apresentadas na Figura 4 mostram que os exemplares do grupo II possuem uma taxa de crescimento mais elevada do que aquelas do grupo I. Nas Tabelas 4-5 verifica-se que tanto $\circ$ incremento em comprimento como em peso, no grupo II, è de aproximadamente três vezes maior; assim, a taxa média de

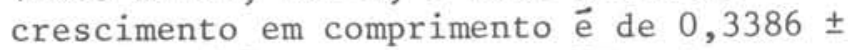
$0,0796 \mathrm{~mm} /$ dia para o grupo II e de $0,1181 \pm 0,0244 \mathrm{~mm} / \mathrm{dia}$ para o grupo I, enquanto que os valores correspondentes ao incremento em peso são, respectivamente, 0,0223 $\pm 0,0048$ gramas/dia e $0,0088 \pm 0,0015$ gramas $/$ dia.

São värios os fatores que influenciam o crescimento de uma espécie, sendo a disponibilidade de alimentos, um deles. Estudando a produção primäria da baía de Santos, região contígua ao Estuário de São Vicente, Gianesella-Galvão (1978), concluiu: "No inverno, os valores varia$\mathrm{ram}$, de 22,6 a $204,2 \mathrm{mgC} \cdot \mathrm{m}^{-3} \cdot \mathrm{h}^{-1} \mathrm{e}$ no verão de 48,0 a $488,4 \mathrm{mgC} \cdot \mathrm{m}^{-3} \cdot \mathrm{h}^{-1}$. Se o número de horas de luz durante o inverno for considerado $11 \mathrm{~h}$, e durante o verão $13 \mathrm{~h}$, então a produção primária para águas de superfície pode variar de 242 a $2354 \mathrm{mgC} \cdot \mathrm{m}^{-3} \cdot \mathrm{dia}{ }^{-1}$ durante o inverno e de 524 a $6344 \mathrm{mgC} \cdot \mathrm{m}^{-3} \cdot \mathrm{dia}^{-1}$ durante o verão (sem levar em conta as variações diurnas)".

\section{Relação peso/comprimento e fator de condição}

Com a anälise dos dados de peso e comprimento pode-se representar as relações peso/comprimento dos grupos I e II pelas seguintes equações:

$\mathrm{W}_{\mathrm{t}}=2,5412 \times 10^{-5} \mathrm{~L}_{\mathrm{t}}{ }^{2,6580} \mathrm{com} \mathrm{r}=0,98$

e $p<0,01$ e

$\mathrm{W}_{\mathrm{t}}=1,0915 \times 10^{-7} \mathrm{~L}_{\mathrm{t}}{ }^{3,9229} \mathrm{com} \mathrm{r}=0,99$

e $p<0,01$, onde $W_{t}$ representa o peso, $L_{t}$ o comprimento $e^{t} \underline{r}$ o coeficiente de correlação linear de Pearson.

Observando-se as curvas apresentadas na Figura 5 verifica-se que atē o comprimento de $73 \mathrm{~mm}$ aproximadamente, os exemplares de mesmo comprimento, do grupo I possuem maior peso que aqueles do grupo
II, invertendo-se a situação para os exemplares maiores.

Quanto ao fator de condição (K) verifica-se inicialmente, pelo exame da Figura 4 e Tabelas 2-3, que os valores encontrados pelos dois métodos utilizados não apresentaram diferenças marcantes entre si, pelo menos no tocante à tendência de seus valores, o que permite considerar-se os valores médios por amostra. A diferença observada certamente é devida ao fato de que no grupo II a taxa de crescimento em comprimento, sendo mais elevada, leva a um menor armazenamento de reservas.

No grupo II é observada uma elevação nos valores de $\overline{\mathrm{K}}$ a partir de maio, época em que o valor do comprimento mëdio situava-se em torno de $70 \mathrm{~mm}$. Para o grupo II o $\overline{\mathrm{K}}$ manteve-se em elevação após atingir $70 \mathrm{~mm}$ de comprimento, enquanto que no grupo I uma acentuada queda é observada de fins de novembro em diante.

Segundo Vazzoler \& Vazzoler (1965) "os valores de $\mathrm{K}$ além de refletirem variações nas condições de nutrição dos indivíduos, podem ser usados para a população adulta como indicador do grau de desenvolvimento sexual, em decorrência do consumo das reservas graxas durante a época de desova".

Neste estudo, uma vez verificada a não dependência com o comprimento dos exemplares, podemos afirmar que a queda observada para os valores de $\overline{\mathrm{K}}$ no fim do período de permanência do grupo I no estuário é devida ao fato de que seus exemplares iniciam o processo de maturação sexual a partir de fins de novembro, aproximadamente. Esta afirmação é corroborada quando confronta-se os dados de estädio de maturidade com os valores observados para o $\overline{\mathrm{K}}$ e verifica-se que todos os exemplares do grupo II apresentavam-se no estádio A (imaturos) enquanto que no grupo I começavam a aparecer indivíduos no estádio B em outubro-novembro, e em dezembro praticamente $100 \%$ dos exemplares encontravam-se neste estádio de maturidade.

A observação dos estádios de maturidade, segundo a escala de quatro estádios utilizada, embora não permita uma análise mais aprofundada do desenvolvimento gonadal, acredita-se ser suficiente para, pelo menos, separar os exemplares que apresentavam-se ou não em processo de maturação. Além disso, segun- 
do Matsuura (1977) a escala de quatro estádios è útil para peixes em que nunca se estudou sua maturação, dada a impossibilidade de classificā-los em estādios mais detalhados, o que ocorre com A. lepidentostole.

\section{Conclusões}

Anchoviella lepidentostole utiliza a região estuarina de São Vicente como uma das áreas de crescimento da espécie.

Após aproximadamente um ano de permanência neste estuário e iniciado o processo de maturação, a espécie deixa a região.

\section{Agradecimentos}

Os autores expressam seu agradecimento ao Sr Saburo Morota por todas as facilidades colocadas à nossa disposição.

\section{Referências bibliográficas}

FIGUEIREDO, J. L. \& MENEZES, N. A. 1978. Manual de peixes marinhos do sudeste do Brasil. II. Teleostei (1). São Paulo, Museu de Zoologia da Universidade de São Paulo, 110p.

GIANESELLA-GALVÃO, S. M. F. 1978. Produção primária da baía de Santos, Estado de São Paulo. Aspectos sobre a eficiência fotossintética num ambiente marinho poluído. Dissertação de mestrado. Universidade de São Paulo, Instituto Oceanogrä́fico. 57p.
HAYASHI, S. \& KONDO. K. 1962. Growth of the Japanese anchovy. I. Seasonal fluctuation in the condition coefficient. Bull. Tokai reg. Fish. Res. Lab., (9):179-191.

LE CREEN,E. D. 1951. The length-weight relationship and seasonal cycle in gonad weight and condition in the perch (Perca fluviatilis). J. Anim. Ecol., 20(2):201-219.

MATSUURA, Y. 1977. O ciclo de vida da sardinha-verdadeira (Introdução à Oceanografia Pesqueira). Publção esp. Inst. oceanogr., S Paulo, (4): $1-146$.

VAZZOLER, A. E. A. de M. 1971. Diversificação fisiológica e morfológica de Micropogon furnieri (Desmarest, 1822) ao sul de Cabo Frio, Brasil. Bolm Inst. oceanogr., S Paulo, 20(2): $1-70$.

1965. Relation between condition factor and sexual development in Sardinella aurita (Cuv. \& Val.). Anais Acad.bras. Ciênc., 37(sup1.):353-359.

(Recebido em 02-fev-1982; aceito em 08-mar-1985) 